\title{
Pulsed-laser-ablation based nanodecoration of multi-wall-carbon nanotubes by Co-Ni nanoparticles for dye-sensitized solar cell counter electrode applications
}

\author{
Alessandra Imbrogno ${ }^{1,2} \cdot$ Rajesh Pandiyan ${ }^{1} \cdot$ Marianna Barberio $^{2} \cdot$ \\ ${\text { Anastasia } \text { Macario }^{3} \cdot \text { Assunta Bonanno }^{2} \cdot \text { My Ali El khakani }}^{1}$
}

Received: 29 March 2017 / Accepted: 4 May 2017/Published online: 12 May 2017

(c) The Author(s) 2017. This article is an open access publication

\begin{abstract}
We report here on the use of pulsed KrF-laser deposition technique (PLD) for the decoration of Multiwall carbon nanotubes (MWCNTs) by Co-Ni nanoparticles (NPs) to form highly efficient counter electrodes (CEs) for use in Dye-sensitized solar cells (DSSC). By varying the number of laser ablation pulses $\left(N_{\mathrm{LP}}=500-60,000\right)$ of the $\mathrm{KrF}$ laser, we were able to control the average size of the Co-Ni NPs and the surface coverage of the MWCNTs by the nanoparticles. The PLD-based decoration of MWCNTs by Co-Ni NPs is shown to form novel counter electrodes, which significantly enhance the power conversion efficiency (PCE) of the DSSCs. Indeed, the DSSCs based on the PLD-decorated $\mathrm{Co}-\mathrm{Ni}$ counter electrodes (obtained at the optimal $N_{\mathrm{LP}}=40,000$ ) are shown to exhibit a PCE value as high as $6.68 \%$, with high short circuit current $\left(J_{\mathrm{sc}}=14.68 \mathrm{~mA} / \mathrm{cm}^{2}\right)$ and open circuit voltage $\left(V_{\mathrm{oc}}=0.63 \mathrm{~V}\right)$. This represents a PCE improvement of $\sim 190 \%$ in comparison to the DSSCs with pristine MWCNTs $(\mathrm{PCE}=2.3 \%)$ and $\sim 7.4 \%$ PCE increase than that of the conventional DSSC made with a sputtered Platinum-based counter electrode. By systematically investigating the local nanostructure of the $\mathrm{Co}-\mathrm{Ni}$ decorated CEs, we found that the Co-Ni NPs layer exhibits a
\end{abstract}

My Ali El khakani

elkhakani@emt.inrs.ca

1 Institut National de la Recherche Scientifique (INRS), Centre Énergie Matériaux et Télécommunications (EMT), 1650 Boulevard Lionel Boulet, Varennes, QC J3X 1S2, Canada

2 Biology, Ecology, and Earth Science Department, University of Calabria, Via Pietro Bucci, 87036 Rende, CS, Italy

3 Environmental and Chemical Engineering Department, University of Calabria, Via Pietro Bucci, 87036 Rende, CS, Italy porous cauliflower-like morphology, of which surface roughness (RMS) is $N_{\mathrm{LP}}$ dependent. Interestingly, both PCE and roughness of the Co-Ni NPs layer are found to exhibit the same $N_{\mathrm{LP}}$ dependence, with a maximum located around the optimal $N_{\mathrm{LP}}$ value of 40,000 . This enabled us to establish, for the first time, a linear correlation between the achieved PCE of DSSCs and the local roughness of their CEs decorated by Co-Ni NPs. Such a correlation highlights the importance of maximizing the surface area of the CoNi coated MWCNTs on the CEs to enhance the PCE of the DSSCs. Finally, Ultra-violet Photoelectron Spectroscopy (UPS) measurements revealed a significant decrease in the local work function $(\Phi)$ of $\mathrm{Co}-\mathrm{Ni}$ NPs decorated MWCNTs based CEs (at $N_{\mathrm{LP}}=40,000, \Phi=3.9 \mathrm{eV}$ ) with respect to that of either pristine MWCNTs $(\Phi=4.8 \mathrm{eV})$ or sputtered-Pt $(\Phi=4.3 \mathrm{eV})$ counter-electrodes. This $\Phi$ lowering of the Co-Ni/MWCNTs based CEs is an additional advantage to enhance the catalytic reaction of the redox couple of the electrolyte solution, and improve thereby the PCE of the DSSCs.

Keywords Pulsed-laser deposition (PLD) · Co-Ni nanoparticles · Nanoparticles/CNTs nanohybrids · UPS · Work function - DSSC counter electrode - Dye sensitized solar cells

\section{Introduction}

Dye sensitized solar cells (DSSCs) are considered to be one of the most promising solar energy conversion devices alternative to the conventional silicon-based photovoltaic (PV) devices due to their facile fabrication process, their relatively low cost of production and a relatively high photoconversion efficiency [1]. A standard DSSC is 
composed of a working electrode (WE) in the form of a thin film of nano-sized $\mathrm{TiO}_{2}$ grains sensitized by a ruthenium-based dye, and of a platinum-based counter electrode (CE). The space between the two electrodes is filled with a redox couple electrolyte; iodide/triiodide $\left(\mathrm{I}_{3}^{-} / \mathrm{I}^{-}\right)$is commonly used in DSSCs. The counter electrode, in particular, is designated to enhance the transfer of electrons between FTO conductive glass and electrolyte solution, improving the redox reaction and, then, the dye regeneration. The material used as counter electrode is dependent on the redox couple used. A thin film of platinum $(\mathrm{Pt})$ deposited on conducting glass is currently used as standard counter electrode for DSSCs, due to its outstanding electrocatalytic properties in the reduction of $\mathrm{I}_{3}^{-}$species and exchange current density [2-5]. The incident photons cause the promotion of excited electron from highest occupied molecular orbital (HOMO) to lowest unoccupied molecular orbital (LUMO) levels of dye molecules. During the deexcitation process, electrons may decay in the conduction band of $\mathrm{TiO}_{2}$ and being transported from photoanode to cathode, where they are transferred from platinum to the electrolyte solution and, due to the redox reaction, they return to the dye molecule. Despite its good properties, the high cost of platinum negatively affects the overall fabrication costs of the DSSCs, limiting thereby their industrial production [6].

In this context, one of the $R \& D$ challenges is to replace the costly platinum electrode with more cost-effective materials having good catalytic ability for the reduction of $\mathrm{I}_{3}^{-}$ions while exhibiting high resistance to corrosion. In this scenario, extensive research has been performed on using carbon-based materials because of their low cost, good electrical conductivity and interesting electrocatalytic properties, which could be further improved through their appropriate decoration with different metal nanoparticles [7-11]. In particular, Nam et al. [12] have shown that by replacing Pt with well-aligned carbon nanotubes (CNTs) as CEs, the conversion efficiency of their DSSCs was $10 \%$ higher than that of standard Pt-based DSSCs. The increase in the cell efficiency was attributed to the large surface area provided by the CNTs and their high electron conductivity. Beside the good electrical properties, CNTs have also a good stability in time, due to their higher corrosion resistance with respect to $\mathrm{Pt}$ electrodes, allowing the CNTsbased CE to maintain unaltered properties of DSSC device for longer periods of time. Koo et al. [13] compared the time-dependent characteristics of some DSSC devices using both CNTs and Pt-based CEs. The former CE showed both higher efficiency and stability during the time without any substantial change of impedance characteristics even after 5 days, while the latter exhibited an increase in the series resistance (by a factor of three). This behaviour may be attributed to the degradation of Pt film and to the consequent loss of adhesion between $\mathrm{Pt}$ and its underlying conductive glass substrate. Carbon nanotubes are, moreover, optimal catalysts for the redox reaction of the electrolyte solution [14-16]. Their high surface area and the presence of defects sites on their functionalized surface enhance the electron transfer and chemical reactivity of these Pt-free CEs. The charge-transport resistance at the electrolyte-CNT interface, in fact, decreases with the increase of CNT loading. One of the critical parameter for the catalysis of the redox reaction is the diameter of the CNTs. Hwang et al. [16] reported that CNTs with larger diameter could improve the reaction rates between the CEs and the electrolyte solution, and have a lower interfacial reaction resistance. On the other hand, hybrid nanomaterials such as CNTs-metal nanoparticles composites [17-23] showed promising photovoltaic properties with an increase in the conversion efficiency with respect to both pristine CNTs and the conventional Pt-based CEs. Zheng et al. [18] reported that the in situ encapsulation of FeNi alloy nanoparticles (NPs) within CNTs, through a calcination process at $600{ }^{\circ} \mathrm{C}$ in $\mathrm{Ar}$ atmosphere and using $\left[\mathrm{Ni}_{2}\right.$ $\left.\mathrm{Fe}(\mathrm{CN})_{6}\right]$ as precursor, enhances the photoconversion efficiency by $\sim 10$ and $180 \%$ with respect to the standard Pt-based CE and the pristine CNT, respectively. Li et al. [20], have also deposited oxidized MWCNTs on FTO via electrophoresis and decorated them with $\mathrm{CoS}$ through electrodeposition, and reported an efficiency enhancements of $\sim 18$ and $240 \%$, with respect to both platinum (PCE of $\sim 5.9 \%$ ) and pristine MWCNTs (PCE of $\sim 2.9 \%$ ) CEs, respectively.

These improvements of the photovoltaic properties of DSSCs through the re-engineering of their CEs are believed to be due to the decoration of carbon-based material with metal NPs that permits to change the work function value of the carbon-based material, obtaining values as closer as possible to the work function of FTOglass [24] and facilitating thereby the transfer of electrons from the FTO to the electrolyte solution. As recently reported in literature [18-20], the decoration of carbon nanotubes with cobalt or nickel compounds, such as $\mathrm{CoS}$ or FeNi, permits to substitute the expensive platinum coating with transition metals without loss of electrocatalytic activity. Indeed, Xing et al. [22] replaced Pt with N-doped CNTs decorated with Nickel and Cobalt NPs obtained from the calcination at $700{ }^{\circ} \mathrm{C}$ of $\mathrm{NiCl}_{2}$ and $\mathrm{CoCl}_{2}$ precursors. Moreover, they have used $\mathrm{H}_{2} \mathrm{SO}_{4}$ (a toxic and hazardous reagent) to remove metal residues on the CNTs' surface. These novel CEs showed good photovoltaic properties, with an increase of $\sim 9 \%$ of efficiency for $\mathrm{Ni}$ decorated CNTs and an increase of $\sim 1 \%$ of efficiency for Co-decorated CNTs with respect to their standard Pt-based CEs $(7.67 \%)$. Even though it is well known that $\mathrm{Co}-\mathrm{Ni}$ alloy 
has good catalytic properties [25] enhancing electron transfer from carbon-based substrate to electrolyte solution [26], their performance depends on the synthesis route and the alloy composition used. In particular, Chen et al. [25] used a mild hydrothermal reduction method to grow $\mathrm{Co}-\mathrm{Ni}$ NPs directly on FTO-glass substrates, and obtained a PCE ( $\eta$ ) of $2.69 \%$ for $\mathrm{Co}_{0.5} \mathrm{Ni}_{0.5}$ nanoparticles which is quite lower than that of standard Pt-based DSSCs. Likewise, Motlak et al. [26] also used a chemical synthesis method to produce Co-Ni NPs by electrospinning at high voltage to deposit them on carbon nanofibres, and obtained a lower PCE value than that of their standard Pt-based DSSC $(5.9 \%)$. This lower PCE value can be probably attributed to the lower defect density in the alloy lattice. Indeed, Chen et al. [25] have clearly shown that the defects which are inherently present in the $\mathrm{Co}-\mathrm{Ni}$ alloy structure plays an fundamental role in the interaction between the $\mathrm{CE}$ and the electrolyte solution. Moreover, the presence of this large amount of defects can provide enormous active sites on the $\mathrm{CE}$ for $\mathrm{I}_{3}^{-}$adsorption and reduction, thereby improving the redox reaction and enhancing the photovoltaic performance of the DSSC device [25]. All these literature results [18-26] suggest that a good control of the crystallinity and morphology of the material is the key to obtain highly performing counter electrodes. Therefore, there is a need to develop a reliable fabrication route that enables the controlled growth of high-crystalline quality of $\mathrm{Co}-\mathrm{Ni}$ nanoparticles to exploit their full electrocatalytic potential in DSSCs.

A variety of chemical and physical methods, including hydrothermal methods [27-32], pyrolysis [18], electrodeposition [20], electrospinning method [14], and calcination [22], have been used for the synthesis of metal NPs and/or their deposition onto several carbon-based materials, such as carbon nanotubes [18, 20, 21], carbon fibres [27, 31] and graphene [28, 29], but most of these techniques require chemical precursors, generally high processing temperatures, long reaction/deposition times (several hours), and most often post-synthesis treatments with strong acids to remove metal residues. As an alternative to these chemical synthesis routes, pulsed-laser deposition (PLD) is a versatile physical method that has been shown to be highly effective for the in situ decoration of different substrates (including CNTs or $\mathrm{TiO}_{2}$ nanorods) by highly pure metallic or semiconducting nanoparticles [33-38] with a fair control over their particle size, surface coverage and crystallinity.

In this paper, we report on the use of the PLD technique to decorate MWCNTs by Co-Ni NPs and form a novel CoNi/MWCNTs nanohybrids to be used as a CE material for DSSCs. To the best of our knowledge, no studies have been reported yet on the PLD-based nanodecoration of MWCNTs by $\mathrm{Co}-\mathrm{Ni}$ nanoparticles for the CE application in DSSCs. In the present study, we have varied the number of laser ablation pulses $\left(N_{\mathrm{LP}}\right)$ of the $\mathrm{Co}-\mathrm{Ni}$ target to control the size of the Co-Ni NPs and the thickness of the $\mathrm{Co}-\mathrm{Ni}$ NPs layer decorating the MWCNTs. We were thus able to identify the optimal $N_{\mathrm{LP}}$ condition that leads to the $\mathrm{Co}-\mathrm{Ni} /$ MWCNTs counter-electrodes exhibiting the highest PCE (of 6.68\%) in our DSSCs. Moreover, the systematic investigation of the roughness of the Co-Ni/MWCNTs nanohybrid CEs has enabled us to establish a correlation between their local roughness to the PCE performance of the DSSCs.

\section{Experimental}

Multi-wall carbon nanotubes (produced by CCVD) used in this study were purchased from Raymor-Nanointegris, with a nominal purity $>99 \%$, an average inner diameter of $4 \mathrm{~nm}$, an average outer diameter $<20 \mathrm{~nm}$, and a length of up to $12 \mu \mathrm{m}$. Prior to use, the MWCNTs were well dispersed by sonicating them in a dimethylformamide (DMF) solution for $2 \mathrm{~h}$, obtaining a suspension with a concentration of $0.3 \mathrm{mg} / \mathrm{mL}$. Uniform films of MWCNTs were then spray-coated onto FTO-glass substrates placed on a hotplate maintained at $200{ }^{\circ} \mathrm{C}$ to facilitate the rapid and complete evaporation of the solvent. The Co-Ni NPs were deposited by means of the PLD technique onto various substrates including the MWCNTs spray-coated films, quartz and silicon wafers. In our PLD process, a $\mathrm{KrF}$ excimer laser $(\lambda=248 \mathrm{~nm}$, pulse duration $=14 \mathrm{~ns}$, repetition rate $=20 \mathrm{~Hz}$ ) beam is focused at an incidence angle of $45^{\circ}$ onto a polycrystalline $\mathrm{Co}-\mathrm{Ni}$ target (commercial $\mathrm{Co}_{0.5} \mathrm{Ni}_{0.5}$ alloy disk of size $2^{\prime \prime}$ in diameter) under a controlled background Helium (He) pressure of 300 mTorr. The substrates were mounted on a rotating substrate holder $(5 \mathrm{rpm})$, and placed parallel to the target at a distance of $6.5 \mathrm{~cm}$ from it during the laser ablation process, to obtain a uniform coverage of the MWCNTs/FTO/glass substrates. The on-target laser energy was set to $140 \mathrm{~mJ} /$ pulse, and the Co-Ni NPs deposition was made as a function of the number of laser pulses $\left(N_{\mathrm{LP}}\right)$, which was varied from 500 to 60,000 pulses. Prior to each deposition, the target surface was cleaned under vacuum for $10 \mathrm{~min}$ while shielding the substrates with a shutter. To fabricate our DSSC devices, the photoanode used was a standard commercially available $\mathrm{TiO}_{2} /$ FTO electrode (Dyesol). The $\mathrm{TiO}_{2}$ was sensitized via immersion in a ruthenium-based dye solution (dye N719, concentration $1 \mathrm{mM}$, Sigma Aldrich) for $24 \mathrm{~h}$. The electrode was rinsed several times with ethanol to remove the excess dye, and then dried in air. Both photoanode and cathode were assembled together as a DSSC device by sealing them using a $25 \mu \mathrm{m}$ thick Surlyn polymer foil 
spacer. The space between working electrodes and counter electrodes was filled with $\mathrm{I}^{-} / \mathrm{I}_{3}^{-}$redox couple electrolyte solution (EL-HPE, Dyesol).

The microstructure and the bulk composition of the $\mathrm{Co}-\mathrm{Ni} /$ MWCNTs nanohybrids (NHs) were examined by STEREOSCAN scanning electron microscope (SEM) working at $20 \mathrm{keV}$ in both Secondary Electron Emission (SE) and Back Scattering (BS) modes. The SEM is equipped with an EDX spectrometer. The surface topography of the $\mathrm{Co}-\mathrm{Ni} /$ MWCNTs nanohybrid CEs was characterized by atomic force microscopy (AFM) measurements using an ICON AFM Microscope (Bruker) in tapping mode and elaborated using Nanoscope software, and their surface roughness (RMS) derived from the AFM images. The chemical bonding states and work function values $(\Phi)$ of the $\mathrm{Co}-\mathrm{Ni} / \mathrm{MWCNTs} \mathrm{NHs}$ were investigated using ex situ X-ray Photoelectron (XPS) and Ultra-violet photoelectron (UPS) spectroscopies, respectively. XPS analyses were performed in ultra-high vacuum (UHV) conditions (with a base pressure of $10^{-9}$ Torr), where a monochromatic $\mathrm{Mg}-\mathrm{K} \alpha \mathrm{X}$-ray (Spectra Instruments, $\mathrm{h} v=1253.64 \mathrm{eV}$ ) was used as excitation source. The $\mathrm{C} 1 \mathrm{~s}$ peak of a pure carbon sample (binding energy position of $284.6 \mathrm{eV}$ ) was used as reference material for the calibration of the XPS spectra. After the Shirley background subtraction, the XPS spectra were fitted using CasaXPS software by adopting Gaussian-Lorentzian peak shapes. UPS measurements were carried out using the $\mathrm{He}$ discharge lamp (He I line, $\mathrm{h} v=21.2 \mathrm{eV}$ ) as the excitation source in normal emission with a resolution of $0.06 \mathrm{eV}$ in the ESCALAB $220 \mathrm{iXL}$ spectrophotometer. A gold reference (having $\Phi=5.0 \mathrm{eV}$ ) was used to calibrate the Fermi level position, applying a $-3 \mathrm{eV}$ bias to eliminate the intrinsic detector work function barrier. The J-V characteristics of the $\mathrm{Co}-\mathrm{Ni} / \mathrm{MWCNTs}$ based DSSC devices were systemically measured in ambient atmosphere under dark and standard illumination condition (AM 1.5) with an incident light power of $100 \mathrm{~mW} / \mathrm{cm}^{2}$ with an Oriel Sol3A solar simulator. The data acquisition was performed using a Keysight B2901A source-meter. Two critical parameters of solar cell performances, Power Conversion Efficiency (PCE) and Fill Factor (FF) were evaluated using the standard equations: $\mathrm{PCE}=\frac{V_{\mathrm{oc}} J_{\mathrm{sc}} \mathrm{FF}}{P_{l}}$ and FF $=\frac{V_{\max } J_{\max }}{V_{\mathrm{oc}} J_{\mathrm{sc}}}$, where $P_{l}$ is the power density of the irradiating light, $V_{\max }$ and $J_{\max }$ are voltage and current density at the maximum power, and $V_{\mathrm{oc}}$ and $J_{\mathrm{sc}}$ are the open circuit voltage and short circuit current density, respectively.

\section{Results and discussion}

Figure 1a, b show typical top-view SEM images of pristine MWCNTs and MWCNTs PLD-coated with $\mathrm{Co}-\mathrm{Ni}$ nanoparticles (at $N_{\mathrm{LP}}=40,000$ ). By comparing the two
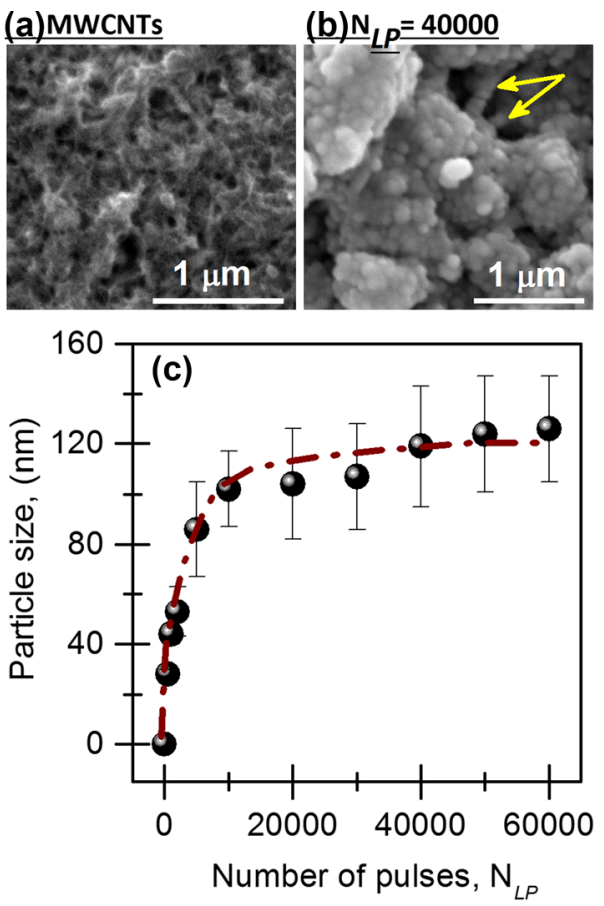

Fig. 1 Typical top-view SEM images of the a pristine MWCNTs and b MWCNTs PLD-decorated with Co-Ni NPs at $N_{\mathrm{LP}}=40,000$; c Variation of average particle size of the Co-Ni NPs PLD-deposited onto the MWCNTs as a function of $N_{\mathrm{LP}}$

images, one can easily note that the Co-Ni NPs have formed a layer that completely wrapped the entangled filamentous structure of the MWCNTs (some coated MWCNTs bundles can still be pinpointed, see the yellow arrows on Fig. 1b). It is also worth noting that the $\mathrm{Co}-\mathrm{Ni}$ NPs decorated MWCNTs exhibit an interesting open and highly porous morphology. By increasing the $N_{\mathrm{LP}}$, the surface coverage of the MWCNTs by $\mathrm{Co}-\mathrm{Ni}$ NPs varies from a partially decorated state (i.e., isolated small $\mathrm{Co}-\mathrm{Ni}$ NPs decorating the MWCNTs for $\left.N_{\mathrm{LP}}=500-20,000\right)$ to a completely covered one (for $N_{\mathrm{LP}} \geq 20,000$ ). On the other hand, the average size of the Co-Ni NPs, at $N_{\mathrm{LP}}=40,000$, is estimated to be of $\sim 110 \mathrm{~nm}$ (Fig. 1b). In fact, the average size of the Co-Ni NPs depends on the number of used laser ablation pulses, as illustrated in Fig. 1c.

Under our PLD conditions, the average diameter of the Co-Ni NPs is found to increase steeply, from $\sim 30$ to $100 \mathrm{~nm}$, when $N_{\mathrm{LP}}$ is increased from 500 to 10,000 , and then slowly increases with a tendency to saturate around an average NP size of $\sim 120 \mathrm{~nm}$ for the highest $N_{\mathrm{LP}}$ values. It is worth noting that as $N_{\mathrm{LP}}$ is increased from 10,000 to 60,000 pulses, the progressive accumulation of $\mathrm{Co}-\mathrm{Ni} \mathrm{NPs}$ onto the bundles of MWCNTs leads to a full coverage of their surface with the formation of sort of cauliflower-like aggregates that may enhance the effective surface of the nanohybrid. However, at the highest $N_{\mathrm{LP}}$ values, the increase of the surface area may be counteracted by the 
coalescence of large aggregates, which will ultimately diminish the open porosity of the CEs. To further examine the surface topography of the Co-Ni NPs layers and estimate their local surface roughness, the AFM technique was systematically used for the different $N_{\mathrm{LP}}$ values. Figure 2ad shows 2D-view of the obtained AFM images for pristine MWCNTs (a) and for MWCNTs PLD-coated with Co-Ni $\mathrm{NPs} \quad$ at $N_{\mathrm{LP}}=20,000 \quad$ (b), $\quad N_{\mathrm{LP}}=40,000 \quad$ (c), and $N_{\mathrm{LP}}=60,000$ (d). Firstly, by comparing the top-view images $(500 \mathrm{~nm} \times 500 \mathrm{~nm}$ scanned area) of Fig. 2, a clear change in the surface morphology of the Co-Ni NPs decorated MWCNTs can be pointed out as $N_{\mathrm{LP}}$ is increased. The most striking aspects are two-fold: (1) the size of the features is clearly seen to increase as $N_{\mathrm{LP}}$ is raised from 20,000 to 60,000 , and (2) the open porosity tends to diminish with increasing $N_{\mathrm{LP}}$, in agreement with our SEM
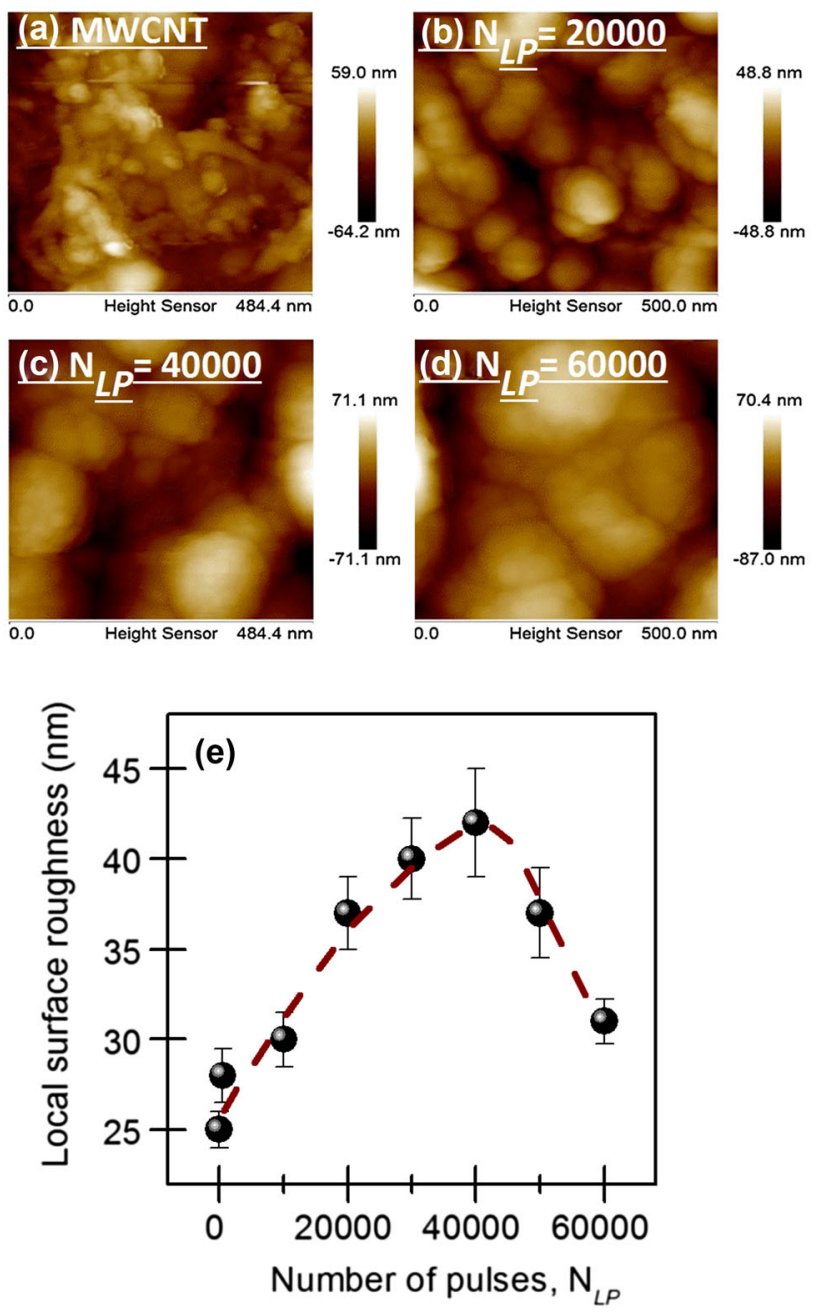

Fig. 2 2D-view of AFM images of a pristine MWCNTs and MWCNTs PLD-coated with Co-Ni NPs layer with b $N_{\mathrm{LP}}=20,000$, c $N_{\mathrm{LP}}=40,000$, d $N_{\mathrm{LP}}=60,000$; e Variation of local surface roughness of the PLD-coated $\mathrm{Co}-\mathrm{Ni}$ NPs layer after their decoration on to the MWCNTs as a function of $N_{\mathrm{LP}}$ observations. In fact, the entangled bundles of MWCNTs (shown in Fig. 2a) are progressively covered with $\mathrm{Co}-\mathrm{Ni}$ NPs that wrap the nanotubes and tend to accumulate as larger agglomerates as a function of $N_{\mathrm{LP}}$ (as shown Fig. 2b-d).

The observed morphology variation of the Co-Ni NPs layers as $N_{\mathrm{LP}}$ is increased can be explained based on the Young's theory [39]: the formation of the aggregates of Co-Ni NPs on the surface of MWCNTs is governed by, on one hand, the nucleation that takes place during the PLD decoration and, on the other hand, the wetting properties of materials involved in the process. The nucleation rate depends on the metal cohesion energy $\left(E_{\mathrm{coh}}\right)$, on the metalsubstrate interfacial energy $\left(E_{\mathrm{f}}\right)$ and on the diffusion barrier ( $\left.E_{\text {diff }}\right)$, while the wetting is regulated by the $E_{\mathrm{f}}$ and by the metal surface energy $\left(E_{\mathrm{s}}\right)$ [39]. It is known that the cluster formation is favoured when $E_{\mathrm{s}}>E_{\mathrm{f}}$, and $E_{\mathrm{diff}}$ has a low value [40]. Moreover, it has been reported that $\mathrm{Co}$ and $\mathrm{Ni}$ NPs synthesized via laser ablation technique in solution, cover uniformly the CNTs with local layer-like growth [41]. This phenomenon is mostly due to the close values of $E_{\mathrm{s}}$ and $E_{\mathrm{f}}$, but also relatively high $E_{\text {diff }}$ of both Co and Ni, that explain the contemporary presence of uniformly dispersed NPs and the local formation of nanoparticle aggregates on the CNTs surface. This particular behaviour of $\mathrm{Co}$ and $\mathrm{Ni}$ and the relatively high number of laser pulses used for the PLD decoration processes may explain the formation of large aggregates at the highest $N_{\mathrm{LP}}$, as revealed by both SEM and AFM observations. To assess quantitatively the observed morphological changes as a function of $N_{\mathrm{LP}}$, the local surface roughness of the Co-Ni NPs deposited onto MWCNTs was determined from corresponding AFM images, as those shown in Fig. 2a-d. Figure 2e shows the variation of local surface roughness $\left(R_{\mathrm{rms}}\right)$ of the PLD Co-Ni NPs layers as a function of $N_{\mathrm{LP}}$, where $R_{\text {rms }}$ is to seen to be $N_{\mathrm{LP}}$ dependent with the presence of a maximum value at $N_{\mathrm{LP}}=40,000$. Indeed, the local surface roughness is found to increase from $25 \mathrm{~nm}$ at $N_{\mathrm{LP}}=0$ (i.e., pristine MWCNTs), to reach its maximum value of $42 \mathrm{~nm}$ for the film decorated at $N_{\mathrm{LP}}=40,000$, and then starts to decrease down to $\sim 31 \mathrm{~nm}$ at $N_{\mathrm{LP}}=60,000$ pulses.

Figure 3 shows the high-resolution core level XPS spectral regions of Ni $2 p$ and Co $2 p$ of the PLD-deposited Co-Ni NPs layers onto MWCNTs, at $N_{\mathrm{LP}}=40,000$. The XPS survey spectra (not shown) confirmed the presence of $\mathrm{Ni}$, Co along with the surface carbon and oxygen contamination. The binding energies (BEs) of all core level regions were corrected using the $\mathrm{C} 1 s \mathrm{BE}$ of $284.6 \mathrm{eV}$. Both Ni $2 p$ and Co $2 p$ core level region shows a complex spectral profile (Fig. 3) containing mixture of core level and intense shake-up satellite signals towards the higher $\mathrm{BE}$ side of the main peak, which can be ascribed to the 


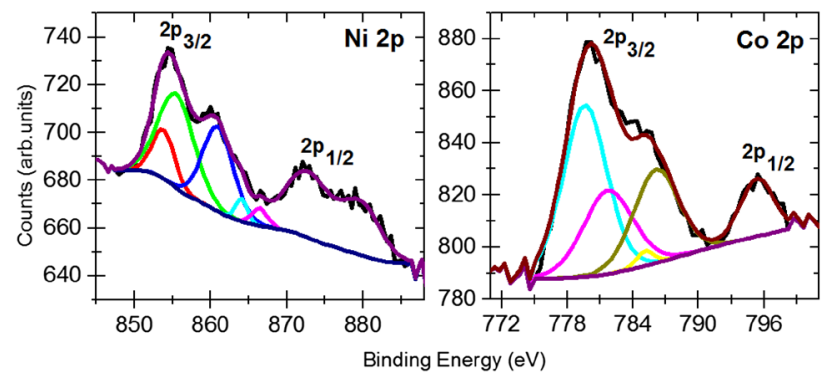

Fig. 3 High-resolution core level XPS spectra of Ni $2 p$ and Co $2 p$ of the Co-Ni nanoparticles layer pulsed-laser-deposited onto MWCNTs, at $N_{\mathrm{LP}}=40,000$

multi-electron excitation processes [42]. For the Nickel, the $\mathrm{Ni} 2 p_{3 / 2}$ peak can be de-convoluted into five peaks contributions centred at the binding energy (BE) position of $853.7 \mathrm{eV}$ along with its associated shake-up satellite peaks at $\mathrm{BE}$ of $855.4,860.9,864.4$ and $866.3 \mathrm{eV}$, in accordance with those reported for $\mathrm{NiO}$ [43]. The $\mathrm{Co} 2 p_{3 / 2}$ region can also be decomposed into four peaks, the main one located at $\mathrm{BE}$ of $779.6 \mathrm{eV}$ and the satellites located at 781.7, 785.1 and $786.1 \mathrm{eV}$, all in agreement with typical XPS peaks of $\mathrm{CoO}$ [43]. Conversely, the $\mathrm{O} 1 \mathrm{~s}$ spectra can be deconvoluted into two different components located at $\sim 529.8$ and $531.9 \mathrm{eV}$. The main peak located at BE $529.8 \mathrm{eV}$ confirms the presence of metal oxygen bonds (i.e., $\mathrm{Ni}-\mathrm{O}$ [42] and $\mathrm{Co}-\mathrm{O}$ [43]), while the less prominent one (at $531.9 \mathrm{eV}$ ) corresponds to the $\mathrm{O}-\mathrm{H}$ bonds of the surface hydroxyl groups [44]. All these results confirm that the outer surface of the Co-Ni NPs is oxidized. This is not surprising since the interaction of $\mathrm{Co}$ and $\mathrm{Ni}$ with the atmospheric oxygen will lead to their surface oxidation.

Interestingly, the presence of $\mathrm{Co}$ and $\mathrm{Ni}$ oxides on the nanoparticles' surface would improve their catalytic activity for the catalysis of iodine-based electrolytes due to the interaction between the ionic feature of $\mathrm{NiO} / \mathrm{CoO}$ and the ions of the electrolyte solution [45]. Moreover, the presence of $\mathrm{CoO}$ layer at the NPs' surface is expected to reduce the transfer resistance of electrons at the interface between the CEs and the electrolyte solution, enhancing thereby the photovoltaic performance of the DSSC device [25]. In complement to XPS surface analyses, EDX measurements were also used to determine the "bulk" composition for all the MWCNTs decorated with Ni-Co NPs. The EDX spectrum (not shown here) confirms the presence of $\mathrm{Co}$ and Ni elements with some minor contributions of $\mathrm{C}$ and $\mathrm{O}$. The average weight percentage of the elemental composition from EDX analysis is found to be Co (47.4\%), $\mathrm{Ni}(46.9 \%), \mathrm{C}(2.2 \%)$ and O (3.5\%). By combining XPS and EDX results, one would suggest that the Co-Ni NPs consist of a metallic core surrounded by an outer $\mathrm{NiO} / \mathrm{CoO}$ oxide shell (of probably few nm-thick, since the detected
XPS photoelectrons typically emerge from a shallow 2-3 nm thick layer).

Prior to assessing the PV performance of our DSSCs devices, we first performed ultra-violet photoelectron spectroscopy (UPS) measurements on the Co-Ni NPs decorated MWCNTs counter-electrodes to see how their work function $(\Phi)$ is affected by the presence of Co-Ni NPs. The determination of $\Phi$ of the CEs is key to access the energy band diagram of the DSSCs. Figure 4a shows a typical UPS spectrum of the Co-Ni/MWCNTs nanohybrid obtained at $N_{\mathrm{LP}}=40,000$, while the inset compares the zoomed-in cutoff region of the secondary electrons spectra for different CEs. The work function $(\Phi)$ can be expressed as, $\Phi$ $(\mathrm{eV})=\mathrm{h} v-\left(E_{\text {cut-off }}-E_{\text {Fermi }}\right)$ [46], where $\mathrm{h} v$ is the energy of the incident light, $E_{\text {cut-off }}$ is the secondary electron emission edge, and $E_{\mathrm{Fermi}}$ represents the Fermi edge. The secondary electron cut-off region can be derived by linear extrapolation of the emission edge to zero intensity. The UPS measurements were calibrated using a gold reference sample, for which a work function value of $5.0 \pm 0.1 \mathrm{eV}$ was correctly obtained in our experimental set up. Moreover, our measured $\Phi$ value for pristine MWCNTs was found to be of $4.8 \mathrm{eV}$, in a satisfactory agreement with literature values [47, 48]. From the inset of Fig. 4a, it is interesting to note that the Co-Ni NPs decoration significantly shifts the cut-off region of the Co-Ni/MWCNTs as a function of the $N_{\mathrm{LP}}$ used (i.e., see the green and red outer spectra corresponding to $N_{\mathrm{LP}}=500$ and 40,000 , respectively). Indeed, the $\Phi$ of MWCNTs/FTO decorated with Co-Ni at $N_{\mathrm{LP}}=500$ is determined to be of $4.7 \mathrm{eV}$, while it decreases to $3.9 \mathrm{eV}$ at $N_{\mathrm{LP}}=40,000$ (identical to the $\Phi \mathrm{Co}-$ $\mathrm{Ni}$ value of $4.0 \pm 0.1 \mathrm{eV}$ measured for different thick PLD Co-Ni films deposited onto quartz or silicon). The observed decrease of $\Phi$ (from 4.7 to $3.9 \mathrm{eV}$ ) as $N_{\mathrm{LP}}$ is increased from 500 to 40,000 can be simply explained by the progressive coverage of the MWCNTs by the Co-Ni NPs. In fact, at low $N_{\mathrm{LP}}$ values, the secondary electrons forming the UPS spectrum originate mainly from the shallow surface of uncovered MWCNTs' (partial coverage by Co-Ni NPs), while they are totally emitted by the Co-Ni NPs that fully cover the underlying nanotubes (at $N_{\mathrm{L}} \geq 20,000$, as observed by SEM). It is worth noting here that the $\Phi$ value $(3.9 \mathrm{eV})$ measured on our Co-Ni/MWCNTs CEs (at $N_{\mathrm{LP}}=40,000$ ) is lower than the work function of $4.3 \mathrm{eV}$, measured on the reference counter-electrodes made of sputter-deposited platinum onto MWCNTs. Such a decreased $\Phi$ value of the Co-Ni decorated MWCNTs/FTO counter-electrodes will favour electrons transfer from the Co-Ni NPs layer to their underlying MWCNTs [49]. To better envision the charge transfer pathways in our DSSCs with the Co-Ni PLD-decorated MWCNTs counter electrodes, Fig. 4b shows the electronic band alignments diagram including all the components of the DSSC. This band energy diagram clearly 
Fig. 4 a Typical UPS spectrum of MWCNTs decorated with a Co-Ni NPs layer at $N_{\text {LP }}=40,000$. The inset compares the zoomed-in secondary electron cut-off region of the UPS spectra for different CEs; and b Schematic band alignment model for the entire DSSC device
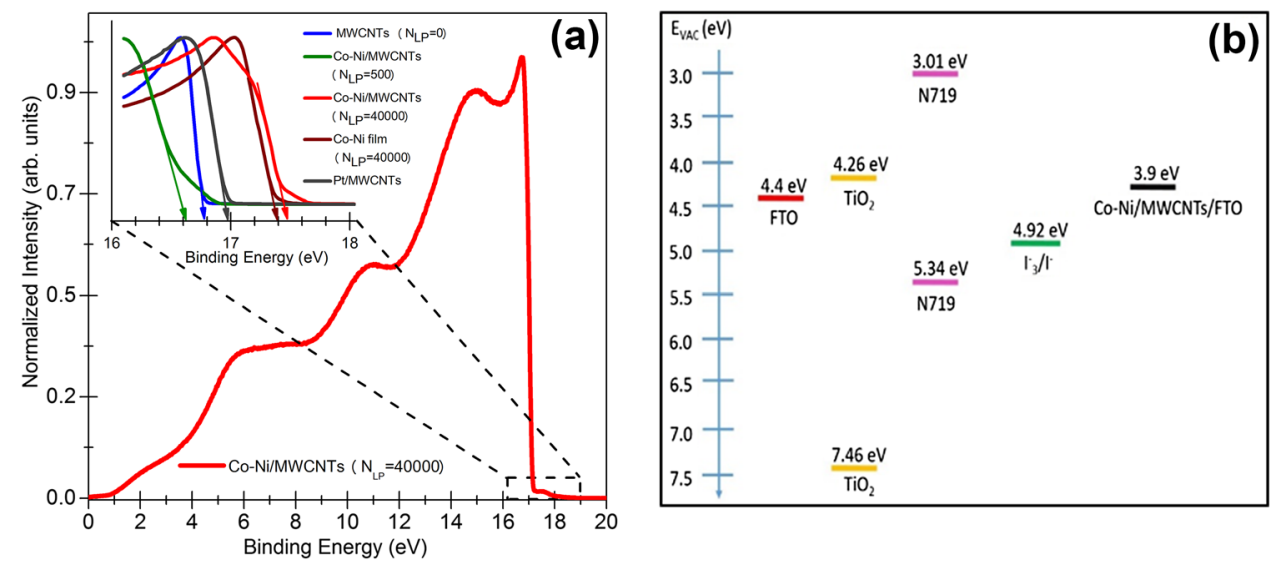

shows that all the energy level positions of individual components are favourable for efficient charge carrier separation and their transfer towards the external electrodes. The absorption of incident photons by the dye molecules causes the promotion of excited electron from the HOMO to the LUMO energy levels. The HOMO and LUMO energy level positions (vs vacuum) of N719 dye are 5.34 and $3.01 \mathrm{eV}$ [50] and the $\mathrm{I}^{-} / \mathrm{I}_{3}^{-}$redox potential is 4.92 [51]. Thanks to the alignment of energy bands of all the components composing the working electrode in a "cascade" structure, the excited electrons from the LUMO band are injected into the conduction band of $\mathrm{TiO} 2$, and then transferred to the FTO substrate (i.e., the working electrode). On the other hand, the electrons that are generated in the photoelectrode are transferred to the cathode (counter electrode) where the aligned energy level positioning allows the flow of electrons from the Co-Ni/MWCNTs/FTO to take part in the reduction of the $\mathrm{I}^{-} / \mathrm{I}_{3}^{-}$species in the electrolyte. The electrons accumulated on the Co-Ni/MWCNTs/FTO counter-electrode are easily exchanged with the electrolyte solution, catalysing thereby the redox reaction that regenerates the dye molecules and enhances the overall photovoltaic performance of the DSSC.

As above-recalled in the introduction, the fundamental role of the CE in DSSC device is to catalyse the redox reaction in the electrolyte solution after the charge injection so that the dye molecules can be regenerated. In this context, the Co-Ni/MWCNTs/FTO (at $N_{\mathrm{LP}}=40,000$ ) exhibiting the lowest local work function $(\Phi=3.9 \mathrm{eV})$ arises as the best counter-electrode which is expected to offer the highest catalytic activity $[49,52]$ among the various $N_{\mathrm{LP}}$ conditions investigated here. The highest porosity and associated surface area observed at $N_{\mathrm{LP}}=40,000$ is an additional feature that would maximize the interaction of the electrolyte with the low work function Co-Ni/MWCNTs/FTO counterelectrode.

Figure 5 shows typical photocurrent density-voltage (JV) characteristics, measured under AM 1.5 calibrated solar simulator, of the DSSCs made with the PLD-decorated Co$\mathrm{Ni} / \mathrm{MWCNTs} / \mathrm{FTO}$ counter electrodes, at different $N_{\mathrm{LP}}$ ranging from 10,000 to 60,000 , while comparing them with reference CEs, namely pristine MWCNTs/FTO (i.e., $\left.N_{\mathrm{LP}}=0\right)$ and a sputter-deposited Pt film (404 nm-thick) onto FTO. It is clearly seen that while the $V_{\mathrm{oc}}$ values for the different DSSCs are comparable, the produced photocurrent $\left(J_{\mathrm{sc}}\right)$ is highly sensitive to $N_{\mathrm{LP}}$ value used to decorate the CE (Fig. 5). Table 1 summarizes the $N_{\mathrm{LP}}$ dependence of the various $\mathrm{PV}$ parameters $\left(V_{\mathrm{oc}}, J_{\mathrm{sc}}, \mathrm{FF}\right.$, and PCE) of the DSSCs made with the Co-Ni NPs decorated CEs.

The DSSCs made with the Co-Ni/MWCNTs CEs are found to exhibit photovoltaic properties superior than those made of pristine MWCNTs or sputtered-Pt reference CEs. Indeed, the $\mathrm{Co}-\mathrm{Ni} / \mathrm{MWCNT}$ s based DSSCs exhibited open circuit voltage $\left(V_{\mathrm{oc}}\right)$ values ranging between 0.60 to $0.66 \mathrm{~V}$, and short circuit current density $\left(J_{\mathrm{sc}}\right)$ increasing from $10.9 \mathrm{~mA} / \mathrm{cm}^{2}$ (at $N_{\mathrm{LP}}=10,000$ ) to a maximum value of $14.7 \mathrm{~mA} / \mathrm{cm}^{2}$ (at $N_{\mathrm{LP}}=40,000$ ). These PV characteristics exceeded those exhibited by DSSCs using a CE made of either pristine MWCNTs (i.e., $N_{\mathrm{LP}}=0$; with $V_{\mathrm{oc}}=0.56 \mathrm{~V}$ and $J_{\mathrm{sc}}=9.8 \mathrm{~mA} / \mathrm{cm}^{2}$ ) or sputtered-Pt film

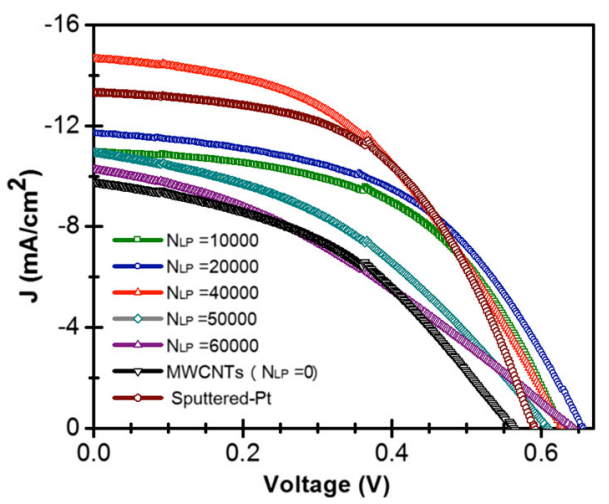

Fig. 5 Typical J-V characteristics, measured under AM 1.5 solar illumination, of the DSSC devices made with pristine MWCNTs, sputtered-Pt and Co-Ni NPs decorated MWCNTs counter electrodes (at different $N_{\mathrm{LP}}$ ranging from 10,000 to 60,000 
Table 1 Photovoltaic parameters of the DSSCs fabricated with different counter electrodes (i.e., pristine MWCNTs, sputtered-Pt, and Co-Ni NPs decorated MWCNTs at different $N_{\mathrm{LP}}$ ), as extracted from the J-V curves of Fig. 5

\begin{tabular}{|c|c|c|c|c|}
\hline Sample & PCE $(\%)$ & $J_{\mathrm{sc}}\left(\mathrm{mA} / \mathrm{cm}^{2}\right)$ & $V_{\text {oc }}(\mathrm{V})$ & $\mathrm{FF}(\%)$ \\
\hline Pristine MWCNTs & 2.30 & 9.80 & 0.56 & 40 \\
\hline $\mathrm{Co}-\mathrm{Ni}\left(N_{\mathrm{LP}}=10,000\right) / \mathrm{MWCNTs}$ & 5.39 & 10.94 & 0.63 & 78.2 \\
\hline $\mathrm{Co}-\mathrm{Ni}\left(N_{\mathrm{LP}}=20,000\right) / \mathrm{MWCNTs}$ & 5.84 & 11.72 & 0.66 & 75.5 \\
\hline $\mathrm{Co}-\mathrm{Ni}\left(N_{\mathrm{LP}}=30,000\right) / \mathrm{MWCNTs}$ & 6.24 & 13.37 & 0.62 & 75.3 \\
\hline $\mathrm{Co}-\mathrm{Ni}\left(N_{\mathrm{LP}}=40,000\right) / \mathrm{MWCNTs}$ & 6.68 & 14.68 & 0.63 & 72.2 \\
\hline Co-Ni $\left(N_{\mathrm{LP}}=50,000\right) / \mathrm{MWCNTs}$ & 4.49 & 10.93 & 0.60 & 68.4 \\
\hline $\mathrm{Co}-\mathrm{Ni}\left(N_{\mathrm{LP}}=60,000\right) / \mathrm{MWCNTs}$ & 4.10 & 10.27 & 0.64 & 62.9 \\
\hline Sputtered-Pt & 6.22 & 13.36 & 0.59 & 78.9 \\
\hline
\end{tabular}

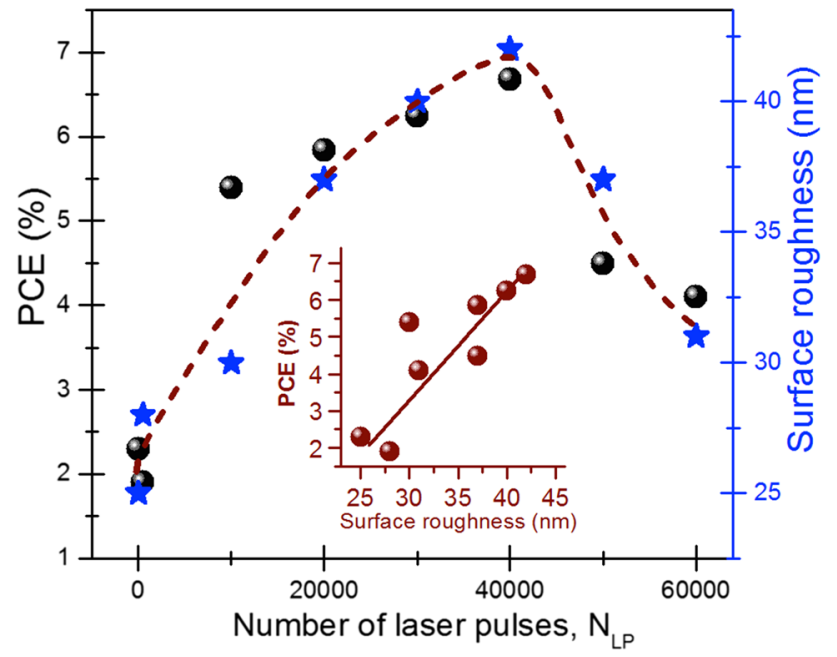

Fig. $6 N_{\mathrm{LP}}$ dependence of both PCE of the DSSCs and surface roughness of their $\mathrm{Co}-\mathrm{Ni} / \mathrm{MWCNTs}$ counter electrodes. The inset shows a fairly linear correlation between PCE of the different DSSCs and the surface roughness of their $\mathrm{Co}-\mathrm{Ni} / \mathrm{MWCNT}$ counter electrodes

reference (with $V_{\mathrm{oc}}=0.59 \mathrm{~V}$ and $J_{\mathrm{sc}}=13.4 \mathrm{~mA} / \mathrm{cm}^{2}$ ). Indeed, the PCE of the DSSCs is shown to increase significantly from $2.3 \%$ at $N_{\mathrm{LP}}=0$ (pristine MWCNTs) to a maximum value of $6.68 \%$ at $N_{\mathrm{LP}}=40,000$, and then progressively decrease down to $4.1 \%$ for the highest $N_{\mathrm{LP}}$ of 60,000 (See Table 1). Thus, $N_{\mathrm{LP}}=40,000$ arises as the optimum PLD decoration condition leading to the highest PCE value of $6.68 \%$, which is $\sim 190 \%$ higher than that of the DSSC made with pristine MWCNTs CEs $\left(N_{\mathrm{LP}}=0\right)$, and $\sim 7.4 \%$ more than that of the sputtered-Pt reference counter electrodes. To better visualize the beneficial effect of the Co-Ni NPs decoration of the MWCNTs CEs, Fig. 6 shows the $N_{\mathrm{LP}}$ dependence of the PCE, where the latter is seen to reach its maximum value of $6.68 \%$ at $N_{\mathrm{LP}}=40,000$. On the same figure, the local surface roughness of the Co-Ni/MWCNTs based CEs is plotted against $N_{\mathrm{LP}}$ (Fig. 6).
Interestingly, both the PCE and the surface roughness of the Co-Ni/MWCNTs CEs are found to show identical $N_{\mathrm{LP}}$ dependence. Indeed, both quantities are seen to increase steadily as $N_{\mathrm{LP}}$ is raised until the optimal value of $N_{\mathrm{LP}}=40,000$, where they reach their respective maxima, after which they markedly decrease for higher $N_{\mathrm{LP}}$ values. Such a similarity suggests that the observed PCE performance of the DSSCs is highly likely linked to the effective surface area of their CEs. Indeed, by cross-plotting the PCE of the DSSCs against the surface roughness of their Co-Ni NPs decorated MWCNTs counter-electrodes, a fairly linear correlation is obtained (inset of Fig. 6). This is the first time that such a direct correlation has been pointed out between the PCE of DSSCs and the surface roughness of their CEs. This clearly indicates that the surface roughness (or more intuitively the effective surface area) of the Co-Ni NPs decorated MWCNTs/FTO counter electrodes has a direct influence on the PCE of the DSSCs. In fact, the maximum PCE observed at $N_{\mathrm{LP}}=40,000$ is thought to result from a synergetic combination of both low work function of the CEs (improvement of catalytic activity through a favored electrons transfer to the electrolyte solution) and their high surface roughness (large effective surface area to interact with the electrolyte). While the low work function of the Co-Ni/MWCNTs CEs is achieved whenever the surface coverage of the MWCNTs by Co-Ni NPs is complete (for $N_{\mathrm{LP}} \geq 20,000$ ), their surface roughness presents a maximum value only around $N_{\mathrm{LP}}=40,000$ (exactly as $N_{\mathrm{LP}}$ dependence of PCE; see Fig. 6). This emphasizes the determining role of the surface roughness of the Co-Ni/MWCNTs based CEs in optimizing the PCE of the DSSCs.

\section{Conclusion}

In summary, we have shown the effectiveness of the physical PLD method to decorate straightforwardly MWCNTs with Co-Ni NPs for the development of novel 
counter electrodes for DSSC applications. These novel Co$\mathrm{Ni} / \mathrm{MWCNTs}$ based CEs were integrated into DSSCs and their devices PCE performance systematically investigated as a function of the $\mathrm{Co}-\mathrm{Ni}$ NPs decoration conditions. Indeed, by varying the $N_{\mathrm{LP}}$ used during the PLD process, we were able to control not only the average $\mathrm{Co}-\mathrm{Ni}$ nanoparticle size, but more importantly the surface coverage rate of MWCNTs by the PLD Co-Ni NPs. Our results revealed that the $N_{\mathrm{LP}}$ variation induces significant changes in the surface roughness of the $\mathrm{Co}-\mathrm{Ni} / \mathrm{MWCNT}$ s counterelectrodes, which in turn are shown to influence directly the overall PV performance of the DSSCs. In fact, the $N_{\mathrm{LP}}=40,000$ is identified as the optimal PLD decoration condition that corresponds at the same time to the highest local surface roughness of the $\mathrm{Co}-\mathrm{Ni} / \mathrm{MWCNTs} \mathrm{CEs}$, and the highest PCE of $6.68 \%$. In a wider picture, we found that both surface roughness of the Co-Ni/MWCNTs CEs and PCE of their associated DSSCs exhibit identical $N_{\mathrm{LP}}$ dependence over the $N_{\mathrm{LP}}=0-60,000$ investigated range. This enabled us to establish, for the first time, a direct linear correlation between the PCE of our DSSCs and the surface roughness of their Co-Ni/MWCNTs counter electrodes. Our results point out the importance of increasing the effective surface area of $\mathrm{Co}-\mathrm{Ni} / \mathrm{MWCNT}$ s counter electrodes for the enhancement of the overall PCE of the DSSCs. The relatively low $\Phi$ value of the $\mathrm{Co}-\mathrm{Ni} /$ MWCNTs (3.9 versus $4.3 \mathrm{eV}$ for the Pt reference CE) is an additional advantage that enhances their catalytic activity through a favored electrons transfer to the electrolyte solution. It is concluded that the PLD decoration of the MWCNTs/FTO counter electrodes by Co-Ni NPs offers an interesting alternative to develop high-performance counter electrodes where the Co-Ni NPs advantageously replace the expensive Pt catalyst commonly used in DSSCs.

Open Access This article is distributed under the terms of the Creative Commons Attribution 4.0 International License (http:// creativecommons.org/licenses/by/4.0/), which permits unrestricted use, distribution, and reproduction in any medium, provided you give appropriate credit to the original author(s) and the source, provide a link to the Creative Commons license, and indicate if changes were made.

\section{References}

1. O’Regan, B., Graetzel, M.: A low cost, high-efficiency solar cell based on dye-sensitized colloidal $\mathrm{TiO}_{2}$ films. Nature 353, 737-740 (1991)

2. Murakami, T.N., Graetzel, M.: Counter electrodes for DSSC: application of functional materials as catalysts. Inorg. Chim. Acta 361, 572-580 (2008)

3. Calogero, G., Calandra, P., Irrera, A., Sinopoli, A., Citro, I., Di Marco, G.: A new type of transparent and low cost counterelectrode based on platinum nanoparticles for dye-sensitized solar cells. Energy Environ. Sci. 4, 1838-1844 (2011)
4. Yin, X., Xue, Z., Liu, B.: Electrophoretic deposition of Pt nanoparticles on plastic substrates as counter electrode for flexible dye-sensitized solar cells. J. Power Sources 196, 2422-2426 (2011)

5. Li, P., Wu, J., Lin, J., Huang, M., Lan, Z., Li, Q.: Improvement of performance of dye-sensitized solar cells based on electrodeposited-platinum counter electrode. Electrochim. Acta 53, 4161-4166 (2008)

6. Wilburn, D.R., Bleiwas, D.I.: Platinum-group metals-world supply and demand, US geological survey open-file report, US department of the interior, Washington (2004). http://www. canplats.ca/images/File/2004-1224.pdf. Accessed 9 Mar 2016

7. Mehmood, U., Ur Rehman, A., Muzammil Irshad, H., Ul Haq Khan, A., Al-Ahmed, A.: Carbon/carbon nanocomposites as counter electrodes for platinum free dye-sensitized solar cells. Org. Electron. 35, 128-135 (2016)

8. Sharma, P., Ahuja, P.: Recent advances in carbon nanotube-based electronics. Mater. Res. Bull. 43, 2517-2526 (2008)

9. Peng, L.-M., Zhang, Z., Wang, S.: Carbon nanotube electronics: recent advances. Mater. Today 17, 433-442 (2014)

10. Kaniyoor, A., Ramaprabhu, S.: Enhanced efficiency in dye sensitized solar cells with nanostructured Pt decorated multiwalled carbon nanotube based counter electrode. Electrochim. Acta 72, 199-206 (2012)

11. Zhang, L., Mulmudi, H.K., Batabyal, S.K., Lam, Y.M., Mhaisalkar, S.G.: Metal/metal sulphide functionalized single-walled carbon nanotubes: FTO-free counter electrodes for dye-sensitized solar cells. Phys. Chem. Chem. Phys. 14, 9906-9911 (2012)

12. Nam, J.G., Park, Y.J., Kim, B.S., Lee, J.S.: Enhancement of the efficiency of dye-sensitized solar cell by utilizing carbon nanotube counter electrode. Scr. Mater. 62, 148-150 (2010)

13. Koo, B.-L., Lee, D.-Y., Kim, H.-J., Lee, W.-J., Song, J.-S., Kim, H.-J.: Seasoning effect of dye-sensitized solar cells with different counter electrodes. J. Electroceram. 17, 79-82 (2006)

14. Lee, W.J., Ramasamy, E., Lee, D.Y., Song, J.S.: Efficient dyesensitized solar cells with catalytic multiwalled carbon nanotube counter electrode. ACS Appl. Mater. Interfaces. 1, 1145-1149 (2009)

15. Trancik, J.E., Barton, S.C., Hone, J.: Transparent and catalytic carbon nanotube films. Nano Lett. 8, 982-987 (2008)

16. Hwang, S., Moon, J., Lee, S., Kim, D.-H., Lee, D., Choi, W., Jeon, M.: Carbon nanotubes as counter electrode for dye-sensitised solar cells. Electron. Lett. 43, 1455-1456 (2007)

17. Huang, K.-C., Wang, Y.-C., Dong, R.-X., Tsai, W.-C., Tsai, K.W., Wang, C.-C., Chen, Y.-H., Vittal, R., Lin, J.-J., Ho, K.-C.: A high performance dye sensitized solar cell with novel nanocomposite film of Pt NP/MWCNT on the counter electrode. J. Mater. Chem. 20, 4067-4073 (2010)

18. Zheng, X., Deng, J., Wang, N., Deng, D., Zhang, W.-H., Bao, X., Li, C.: Podlike N-doped carbon nanotubes encapsulating FeNi alloy nanoparticles: high performance counter electrode materials for dye-sensitized solar cells. Angew. Chem. Int. Ed. 53, 7023-7027 (2014)

19. Xiao, Y., Wu, J., Lin, J.-Y., Tai, S.-Y., Yue, G.: Pulse electrodeposition of $\mathrm{CoS}$ on MWCNT/Ti as a high performance counter electrode for a Pt-free dye-sensitized solar cell. J. Mater. Chem. A 1, 1289-1295 (2013)

20. Li, J.-Y., Liao, J.-H., Hung, T.-Y.: A composite counter electrode of CoS/MWCNT with high electrocatalytic activity for dye sensitized solar cells. Electrochem. Commun. 13, 977-980 (2011)

21. Yue, G., Wu, J., Lin, J.-Y., Xiao, Y., Tai, S.-Y., Lin, J., Huang, M., Lan, Z.: A counter electrode of multi wall carbon nanotubes decorate with tungsten sulfide used in dye-sensitized solar cell. Carbon 55, 1-9 (2013)

22. Xing, Y., Zheng, X., Wu, Y., Li, M., Zhang, W.-H., Li, C.: Nitrogen-doped carbon nanotubes with metal nanoparticles as 
counter electrode materials for dye sensitized solar cells. Chem. Commun. 51, 8146-8149 (2015)

23. Anouar, M., Jbilat, R., Le Borgne, V., Ma, D., El Khakani, M.A.: Ag nanoparticle-decorated single wall carbon nanotube films for photovoltaic applications. Mater. Renew. Sustain. Energy 5, 1 (2016)

24. Shi, Y., Kim, K.K., Reina, A., Hofmann, M., Li, L.-J., Kong, J.: Work function engineering of graphene electrode via chemical doping. ACS Nano 4, 2689-2694 (2010)

25. Chen, X., Tang, Q., He, B., Lin, L., Yu, L.: Platinum-free binary Co-Ni alloy counter electrodes for efficient dye-sensitized solar cells. Angew. Chem. 53, 10799-10803 (2014)

26. Motlak, M., Barakat, N.A.M., Akhtar, M.S., Hamza, A.M., Kim, B.-S., Kim, C.S., Khalil, K.A., Almajid, A.A.: High performance of NiCo nanoparticles-doped carbon fibers as counter electrode for dye-sensitized solar cells. Electrochim. Acta 160, 1-6 (2015)

27. Rameez, Md, Saranya, K., Subramania, A., Sivasankar, N., Mallik, S.: Bimetal (Co-Ni) nanoparticles-incorporated electrospun carbon nanofibers as an alternative counter electrode for dye-sensitized solar cells. Appl. Phys. A 122, 1-10 (2016)

28. Yue, G., Yang, G., Li, F., Wu, J.: PEDOT: PSS assisted preparation of a graphene/nickel cobalt oxide hybrid counter electrode to serve in efficient dye-sensitized solar cells. RSC Adv. 5, 100159-100168 (2015)

29. Du, F., Zuo, X., Yang, Q., Li, G., Tang, H., Zhang, H., Wu, M., $\mathrm{Ma}, \mathrm{Y}$.: The stabilization of $\mathrm{NiCo}_{2} \mathrm{O}_{4}$ nanobelts used for catalysing triiodides in dye-sensitized solar cells by the presence of RGO sheets. Sol. Energy Mater. Sol. Cells 149, 9-14 (2016)

30. Khoo, S.Y., Miao, J., Yang, H.B., He, Z., Leong, K.C., Liu, B., Tan, T.T.Y.: One-step hydrothermal tailoring of $\mathrm{NiCo}_{2} \mathrm{~S}_{4}$ nanostructures on conducting oxide substrates as an efficient counter electrode in dye-sensitized solar cells. Adv. Mater. Interfaces 2, 1500384 (2015)

31. Chen, L., Zhou, Y., Dai, H., Yu, T., Liu, J., Zhou, Z.: One-step growth of $\mathrm{CoNi}_{2} \mathrm{~S}_{4}$ nanoribbons on carbon fibers as platinum-free counter electrodes for fiber-shaped dye-sensitized solar cells with high performance: polymorph-dependent conversion efficiency. Nano Energy 11, 697-703 (2015)

32. Liu, J., Li, C., Zhao, Y., Wei, A., Liu, Z.: Synthesis of $\mathrm{NiCo}_{2} \mathrm{~S}_{4}$ nanowire arrays through ion exchange reaction and their application in Pt-free counter-electrode. Mater. Lett. 166, 154-157 (2016)

33. Ka, I., Ma, D., El Khakani, M.A.: Tailoring the photoluminescence of PbS-nanoparticles layers deposited by means of the pulsed laser ablation technique. J. Nanopart. Res. 13, 2269-2274 (2011)

34. Dai, Q., Chen, J., Lu, L., Tang, J., Wang, W.: Pulsed laser deposition of CdSe quantum dots on $\mathrm{Zn}_{2} \mathrm{SnO}_{4}$ nanowires and their photovoltaic applications. Nano Lett. 12, 4187-4193 (2012)

35. Ka, I., Le Borgne, V., Ma, D., El Khakani, M.A.: Pulsed laser ablation based direct synthesis of single wall carbon nanotubes/ $\mathrm{PbS}$-QDs nanohybrids exhibiting strong, spectrally wide and fast photoresponse. Adv. Mater. 24, 6289-6294 (2012)

36. Ka, I., Gonfa, B., Le Borgne, V., Ma, D., El Khakani, M.A.: Pulsed laser ablation based synthesis of PbS-quantum dots decorated one-dimensional nanostructures and their direct integration into highly efficient nanohybrid heterojunction-based solar cells. Adv. Funct. Mater. 24, 4042-4050 (2014)
37. Gautier, L.A., Le Borgne, V., Delegan, N., Pandiyan, R., El Khakani, M.A.: Field electron emission enhancement of graphenated MWCNTs emitters following their decoration with $\mathrm{Au}$ nanoparticles by a pulsed laser ablation process. Nanotechnology 26, 045706 (2015)

38. Fujisawa, K., Ka, I., Le Borgne, V., Kang, C.-S., Kobayashi, K., Muramatsu, H., Hayashi, T., Kim, Y.A., Endo, M., Terrones, M., El Khakani, M.A.: Elucidating the local interfacial structure of highly photoresponsive carbon nanotubes/PbS QDs based nanohybrids grown by pulsed laser deposition. Carbon 96, 145-152 (2016)

39. He, Y., Zhang, J., Wang, Y., Yu, Z.: Coating geometries of metals on single-walled carbon nanotubes. Appl. Phys. Lett. 96, 063108 (2010)

40. Scarselli, M., Camilli, L., Castrucci, P., Nanni, S.F., Gobbo, D., Gautron, E., Lefrant, S., Crescenzi, M.D.: In situ formation of noble metal nanoparticles on multiwalled carbon nanotubes and its implication in metal-nanotube interactions. Carbon 50, 875-884 (2012)

41. Barberio, M., Stranges, F., Xu, F.: Coating geometry of Ag, Ti $\mathrm{Co}, \mathrm{Ni}$ and $\mathrm{Al}$ nanoparticles on carbon nanotubes. Appl. Surf. Sci. 334, 174-179 (2015)

42. McIntyre, N.S., Cook, M.G.: X-ray photoelectron studies on some oxides and hydroxides of cobalt, nickel and copper. Anal. Chem. 47, 2208-2213 (1975)

43. Biesinger, M.C., Payne, B.P., Grosvenor, A.P., Lau, L.W.M., Gerson, A.R., Smart, R., St, C.: Resolving surface chemical states in XPS analysis of first row transition metals, oxides and hydroxides: Cr, Mn, Fe, Co and Ni. Appl. Surf. Sci. 257, 2717-2730 (2011)

44. Hassel, M., Freund, H.-J.: High resolution XPS Study of a thin $\mathrm{CoO}$ (111) film grown on Co (0001). Surf. Sci. Spectra 4, 273-278 (1990)

45. Wang, H., Wei, W., Hu, Y.H.: $\mathrm{NiO}$ as efficient counter electrode catalyst for dye-sensitized solar cells. Top. Catal. 57, 607-611 (2014)

46. Gutmann, S., Wolak, M.A., Conrad, M., Beerbom, M.M., Schlaf, R.: Effect of ultraviolet and X-ray radiation on the work function of $\mathrm{TiO}_{2}$ surfaces. J. Appl. Phys. 107, 103705 (2010)

47. Shiraishi, M., Ata, M.: Work function of carbon nanotubes. Carbon 39, 1913-1917 (2001)

48. Liu, P., Sun, Q., Zhu, F., Liu, K., Jiang, K., Liu, L., Li, Q., Fan, S.: Measuring the work function of carbon nanotubes with thermionic method. Nano Lett. 8, 647-651 (2008)

49. Deng, D., Yu, L., Chen, X., Wang, G., Jin, L., Pan, X., Deng, J., Sun, G., Bao, X.: Iron encapsulated within Pod-like carbon nanotubes for oxygen reduction reaction. Angew. Chem. Int. Ed. 52, 371-375 (2013)

50. Zhang, J., Yu, C., Wang, L., Li, Y., Ren, Y., Shum, K.: Energy barrier at the $\mathrm{N} 719$-dye/CcCnI 3 interface for photogenerated holes in dye-sensitized solar cells. Sci. Rep. 4, 6954 (2014)

51. Cariello, M., Ahn, S., Park, K.-W., Chang, S.-K., Hong, J., Cooke, G.: An investigation of the role increasing pi-conjugation has on the efficiency of dye-sensitized solar cells fabricated from ferrocene-based dyes. RSC Adv. 6, 9132-9138 (2016)

52. Ni, S., Li, Z., Yang, J.: Oxygen molecule dissociation on carbon nanostructures with different types of nitrogen doping. Nanoscale 14, 1184-1189 (2012) 